\title{
INTRODUCTION TO THE PROTOTYPING OF AN INTELLIGENT SUPERVISION SYSTEM
}

\author{
Samuel Bassetto ${ }^{1,2}$, Ali Siadat ${ }^{1}$, Patrick Martin ${ }^{1}$ \\ ${ }^{1}$ LGIPM-ENSAM, 4 rue Augustin Fresnel, 57050 Metz, France \\ ${ }^{2}$ SYNLOG, 16 Chemin de Malacher, ZIRST 4402, 38944 Grenoble, France \\ samuel.bassetto, \{ali.siadat, patrick.martin\}@metz.ensam.fr
}

\begin{abstract}
This paper introduces the prototyping of an intelligent supervision system, intended to become an advanced manufacturing process control system, and is a summary of its conceptual framework. Constraints linked to each development on an industrial field let emerge new concepts and in our case the two parts of this framework: the structure of the advanced manufacturing process control system and the advanced application development methodology. Their instantiations to our industrial study-case let us prove their validity by solving environmental constraints and exploiting industrial and scientific issues.
\end{abstract}

\section{INTRODUCTION}

Industrial development areas are rich of scientific issues and potential innovations, but they contain a lot of barriers for this richness exploitation.. Among others, the acceptability constraint must not be underestimated, so as being able to explore industrial and scientific issues. A balance between knowledge and technology must be at the heart of industrial scientific developments so as exploring industrial potential and reasoning on impacts of such exploitations. In accordance with such an argument, we followed an innovation process explained in [PEUGEOT PSA CITROEN, 1999], which led us to the development of a conceptual framework for the construction of an advanced manufacturing process control system. Its declination to our scenario application, allowed us to exceed some industrial constraints and build a prototype of an intelligent process supervision system. The scope of this paper is to give in the first chapter a summary of this conceptual framework and in the second chapter to introduce the prototyping of the intelligent process supervision system through its development and its results. 


\section{CONCEPTUAL FRAMEWORK SUMMARY AND ITS DISPLAYS}

While taking as a starting point [Avenier, 1989] our research can be viewed as an intervention and an integration research. The conceptual framework aims to fulfill the need of an advanced manufacturing process control system, its usability and acceptability in an industrial context.

\subsection{Conceptual Framework Summary}

To answer at those requirements, we make the distinction in the framework between "What is to do?" which explains basis concepts to be implemented to achieve this goal and "How to do it?" which explains the implementation process.

The conceptualization operations used for the development of this framework means shed light on concepts, their articulations and the study of their feasibility. This feasibility is studied either a-priori with a full bibliography or a-posteriori with tests implementing concepts and their articulations.

The conceptual framework is composed of two main parts: the architecture of an advanced manufacturing process control system and the advanced iterative collaborative prototyping.

\subsubsection{Conceptualization of the advanced process control system}

The framework, shown Figure1, underlines the four main parts which are the heart of the advanced manufacturing process control system. This system is built in the following order: the transmitting and collecting data module, the analysis and case base module, the control learning module, and the knowledge retroaction module. It is plugged over the production system, from which it retrieves data and to which it gives orders or advices. At the end of the instantiation process users can access to a set of off line tools and to a set of on line tools.

\subsubsection{Conceptualization of processes used to exceed industrial usability and acceptability constraints}

The incremental methodology proposed by [Jacqueson and al., 2001] combined with the collaborative prototyping approach explained in [Terwiesch and Loch, 2002] helped us in the conceptualization of a methodology used to answer at the "how to do it ?" question. 


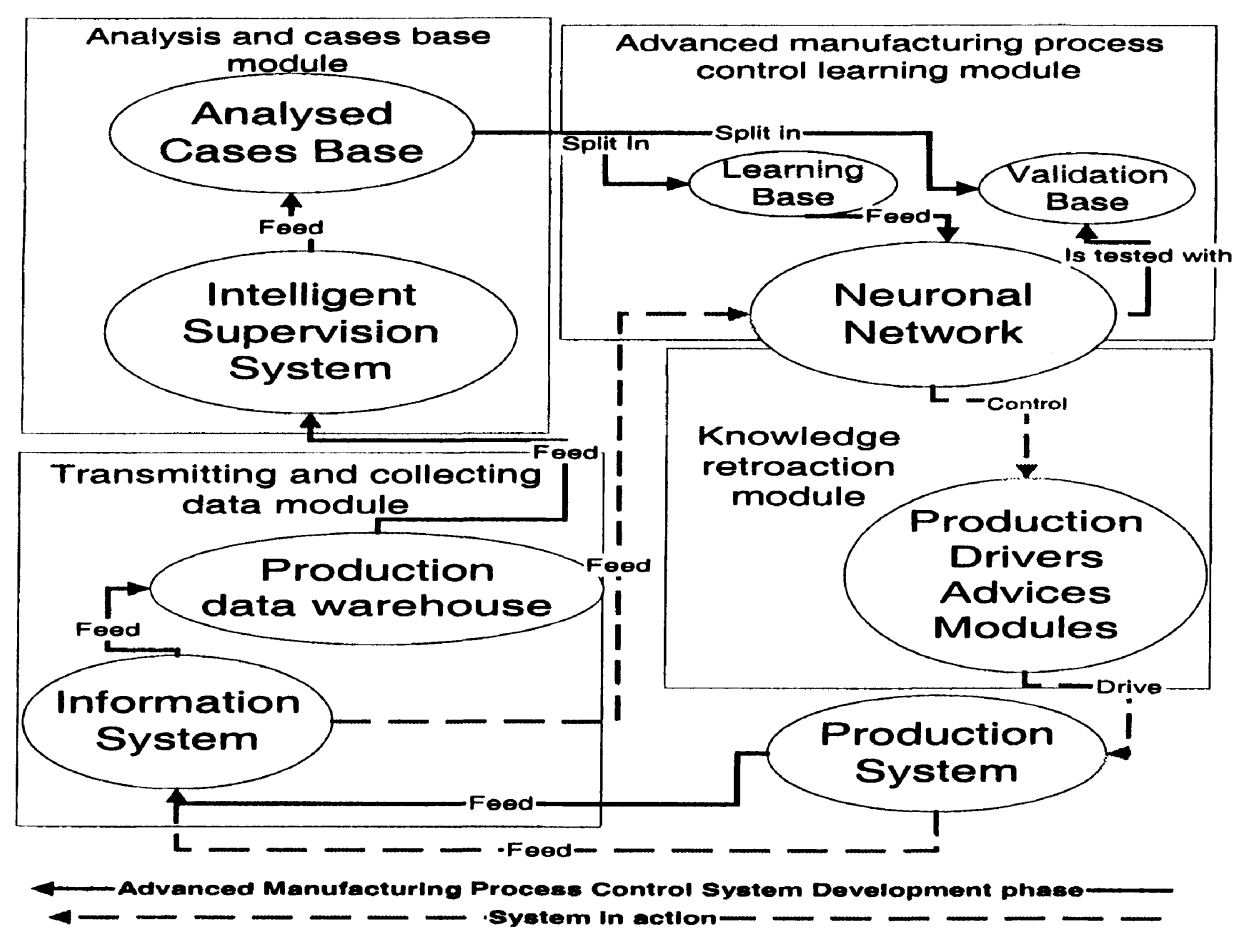

Figure 1- Advanced Manufacturing Process Control System Conceptualization

Detailed Figure 2, two approaches are used which look at the industrial problem in different manners in order to be able to emphasize users needs easily. A development phase follows the discovering of functionalities. The iteration of this circuit leads to the rapid development of a prototype containing a large part of the functionalities needed by users.

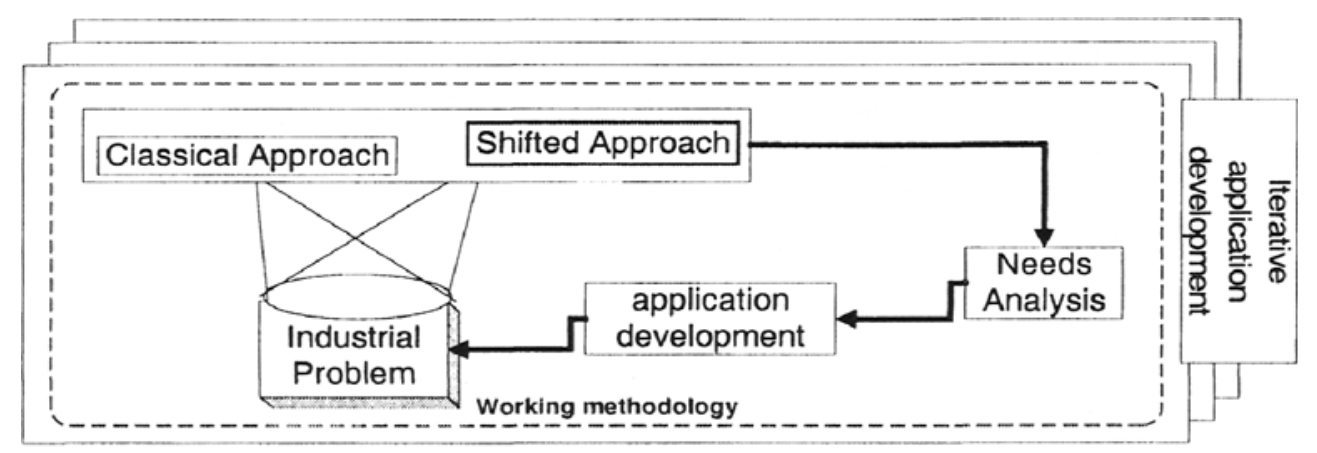

Figure 2 - Advanced Collaborative Prototyping Conceptualization

\subsection{Declination of this Conceptual Framework to our Study Case}

Once the conceptual framework has been created, its feasibility must be tested. This is done by a-priori and a-posteriori ways. This framework is instantiated to a 
particular industrial case. We pay a particular attention to the intelligent supervision system. We identify four major constraints to solve with the advanced collaborative prototyping approach:

- A resource constraint: a little programming background is required and each development is done using basic tools.

- A fixed development time: seven months have been allocated for the specific system development. This constraint was not extendable.

- A goal constraint: software, which is stable when you follow the procedure, must answer user's specifications at the end of the development time (USABILITY CONSTRAINT).

- Utility goal: We focus our approach on the important point of acceptability and the fixed goal of building an accepted tool (UTILITY CONSTRAINT).

The classical approach is the user's specifications analysis while the shifted approach is a knowledge management one, $\mathrm{MKSM}^{1}$, detailed in [Ermines, 1996] and [Ermine \& al. 1996]. This emphasized approach is, explained at and chosen with, the final user. Other shifted approach could have been chosen. In particular, the CIM-OSA ${ }^{2}$ methodology [Vernadat, 1996] could have been used as a guideline into the complex world, in the sense of [Morin, 1990], of enterprise and specifications understanding. The reason for choosing the MKSM approach is explained further in the text.

\section{PROTOTYPING AND RESULTS}

The prototyping of the intelligent supervision system is, as mentioned before, a crucial piece of the advanced process control system. It hangs on an existing process datawarehouse. It is built in a continuous flow production.

\subsection{Aspects of the Prototyping Steps}

The prototype, Figure 3, is composed of five modules which each provide different functionalities. The intelligent supervision system finds its data into a process data warehouse, built for collecting and organizing process sampled signals. This construction comes from industrial goals and groundwork.

\footnotetext{
' Methodology for Knowledge System Management

${ }^{2}$ Computer Integrated Methodology - Open System Architecture
} 


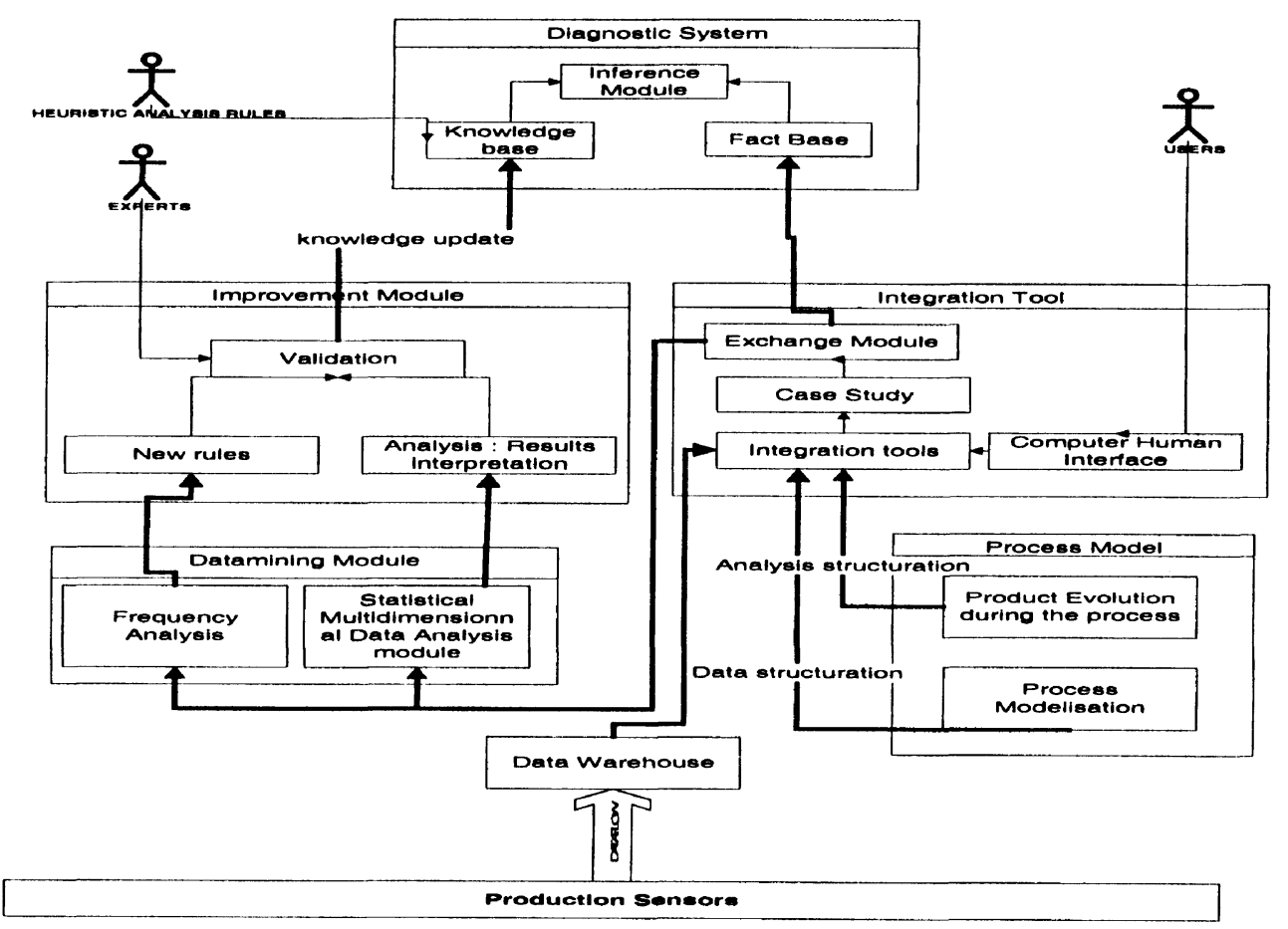

Figure 3 - Intelligent Supervision System for a Manufacture Process Default Analysis Prototype

\subsubsection{Industrial Scenario and Groundwork}

On one hand a data warehouse was still built and there wasn't any powerful exploitation of it. On the other hand, process experts possess a precise knowledge of the manufacture but without a formalization of it. Moreover, no express knowledge management methodology was used to capitalize on projects and individual experiences. [Faure \& Bisson, 2000] and [Ermine \& al.1996] the MKSM knowledge management approach for the shifted view.

An important problem is that data warehouse exploration algorithms like those explain in [Szymkowiak \& al., 2001] or in [Agrawal \& Srikant, 2000] and knowledge reuse system like classical expert system as CLIPS ${ }^{3}$, JESS $^{4}$, CLASSIC $^{5}$ etc are really incompatible. $\mathrm{PCA}^{6}$, clustering algorithms [Sudipto, G. and al. 1998] and frequency analysis requires a wide range of data to discover some relevant information and knowledge. At the opposite, cases based systems and rules systems require a small amount of filtered data and a large scale of formalized knowledge as explained in [CLIPS, 1998] and [Napoli, 1997]. Algorithms used for mining data and those used for knowledge manipulation are different and can not be swapped. However those systems are useful for solving industrial needs (for example): capitalizing on process knowledge and discovering new process rules. The

\footnotetext{
${ }^{3} \mathrm{C}$ Language Integrated Production System

${ }^{4}$ Java Expert System Shell

${ }^{5}$ CLASSIC is a Knowledge Representation language.

${ }^{6}$ Principal Component Analysis
} 
intelligent supervision system fills in this lack by providing a solution able to use expert system and to find and reuse the new rules as shown in 3.2.

The intelligent supervision system prototyping hangs on an existing process data warehouse which aims to store all sampled process variables able to be retrieve through the information system. Its design is similar than those explained in [Lefébure \& al., 2001]. Before being stored in the data warehouse, data are rudimentary compressed, in order to reduce their size. With this technique one day of data is a $\sim 200 \mathrm{MB}$ sized base. This rudimentary compression can be improved. Therefore the goal of this data warehouse is to be implemented in an elementary manner on very simple and inexpensive tools in order to be quickly operational and to save the larger amount of process data. We find again the necessity of solving industrial constraints underlined above. This data warehouse is done to store pairs (Time, Value) of each process variable able to be retrieved through the information system. Each table containing those couples, contain also the date of their recording, the correspondent variable, its dimension, and commentaries on this variable. Tables are indexed by a unique table name correlated to the measurement name. A table can be represented by the vector (VariableName; Measuresdimension; Date;Commentary;(Time1,Value1);...;(Time LimitorTheTableLength, $_{\text {Value }}$ LimitOfTheTableLength $)$

\subsubsection{Prototyping the Integration Tool}

The integration tool module is the heart of the prototype. We can see its importance throughout its development phases.

- In the first construction step, it retrieves basically user's relevant data from the data warehouse and shows them to users.

- In the second development step, it integrates a physical process model to analyze basically some of the user's relevant data and deliver its analysis. This entity-relationship model leads to the formalization of process variables and their physical implantation.

- In the third step, it integrates a model of the product evolution during its manufacture, which allows the tool to make a complete case study with the user's relevant data. This model, combined with the use of a data warehouse, bring some innovations detailed in the next paragraph. At this level the integration tool possess an advanced Computer Human Interface.

- In the last development cycle, exchange modules are conceived in order to build an integrated system with other modules.

The integration is done by exchanging, between modules, well known information transformed or created for this purpose from data. Four validation steps enroll users for using the developed tool and achieving the software acceptability goal. This acceptation process is fully discussed in [Beauvois \& al., 1987]. The main part of 
usability constraint is achieved; this module answers basic users' questions. Its construction is detailed in [Bassetto, 2001].

\subsection{Prototype Main Contributions and Limitations}

\subsubsection{Integration Tool}

Implemented in $\mathrm{VB}$, using the limited, but fully spread into the firm, database engine ACCESS ${ }^{\circledR}$ and following some specific models, the integration tool disconnects the data warehouse from data preprocessing steps and data treatment parts. With its models, the integration tool, picks data up from the data warehouse and applies different algorithms on retrieved data. Either data can be preprocessed for a wider analysis, with some statistical algorithms as detailed below, or data can be filtered with particular production models to obtain users relevant information and feed an expert system. So, the integration tool allows the coexistence of those two ways of thinking.

\subsubsection{Product/Process Model}

Product quality tests are provided at the end of the manufacturing process. If a particular failure is detected, process experts have to find the position where the default occurs for the first time. This is empirically done by process experts who follow the product in the inverse sense of its manufacture evolution. Each sensor encountered during this evolution is tested. However experts look at sensors' values during a fixed temporal window of $\mathrm{M}$ minutes before the event measurement. Let note: $t_{\text {event }}$ is the time where the default is measured and $X$ is a number of seconds, $\mu_{\text {Measurement }}$ is the mean of a specific measurement and $\sigma_{\text {Measurement }}$ its standard deviation. In order to find the default origin process experts look at the variation of each sensor during the $\left[\mathrm{t}_{\text {event }}-\mathrm{X} ; \mathrm{t}_{\text {event }}\right]$ period. They infer that the focused sensor "sees" something if some outsider measures are detected. An outsider is a measure which is not into the interval $\left[\mu_{\text {Measurement }}-3 \sigma_{\text {Measurement }} ; \mu_{\text {Measurement }}+3 \sigma_{\text {Measurement }}\right]$. This way of analyzing signals is not satisfactory. The product evolves in the plant during its production. If we want to find the default origin, we must look at each sensor value at the time when the part of the product analyzed containing the default was in front of the sensor. Only at this time, if an outsider is detected, we can infer a probable source of default as shown Figure 4., where no outsiders are detected!

The process can be modelized by Figure5., and the follwing equations: the product speed between Xe \& Xs, which is : $V x=V(x)=a * x+b$, where a \& b are determined by the line geometry and product Velocity. We measure, $\mathrm{V}_{\mathrm{Xs}}=\mathrm{V}_{\text {out }}$, $\mathrm{V}_{\mathrm{Xe}}=\mathrm{V}_{\text {in }}$. Xe, $\mathrm{Xs}, \mathrm{X}_{\text {obs, }} \forall \mathrm{i}$ Xsensor ${ }^{\mathrm{i}}$ is known with industrial plans, then if a default is detected at $t_{\text {event }}$, then it was in front of sensor ${ }^{i}$ at $t_{\text {sensor }}^{i}$ : 

O)-Vs/a) $\left.\left.) * a / V_{X s}\right)\right)-\left(X_{e}-X_{\text {sensor }}^{i}\right) / V_{\text {in. }}$

This is the model use to analyze accurately data. It is implemented in the integration tool. This operation of retrieving important signals can be a form of filtering. Analyzing the process by this way, noises around relevant process experts information are heavily reduced and only important information are coming out this filter. The case study operation is done more quickly with this model embedded, than without. This operation is more accurate than its correspondent realized by process experts with their analysis method. They are not able to determine exactly if a noised signal contain or not outsiders.

This basic Product/Process model is used here to achieve concepts validation. It could be developed in order be more accurate in the process analysis and to allow a kind of process simulation.

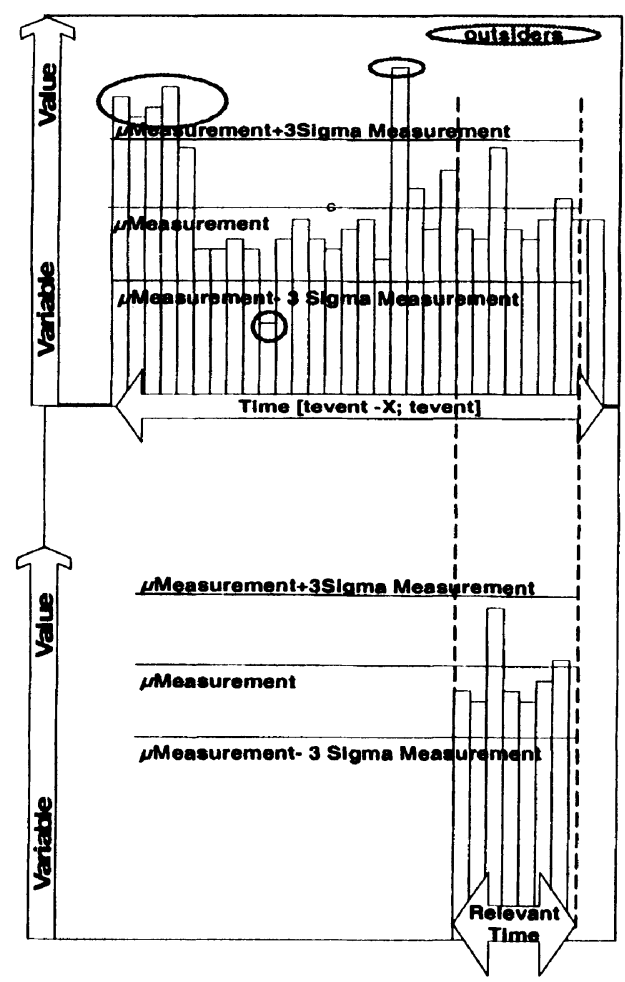

Figure 4. Relevant Values without Outsiders

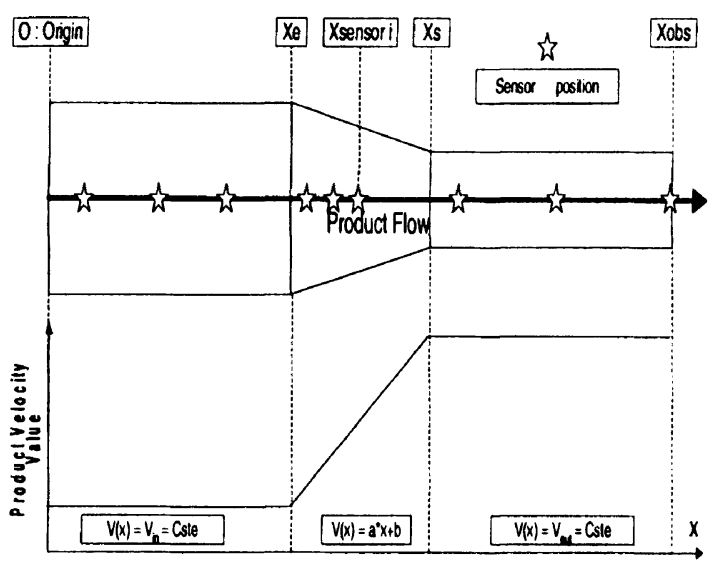

Figure 5 - Physical Line Model. 


\subsubsection{Datamining and Its Integration}

To underline the contribution of the datamining module, the following example is considered. Starting with 150 variables for a relevant part of the process with a sampling rate of 1 sample per second, we apply a PCA algorithm and reduce the dimension space from 150 to 8 , we project all data onto the two more significant variables and a result among others, as shown in Figure 6, underlines that no trend can be observed from a stationary zone to an unstable one.

Before this analysis the knowledge base contains, among others, the following rule: If A DEFAULT IS MEASURED then LOOK AT ALL PROCESS TRENDS. This process rule was based on expert routine experiences to looking at any sensors trends. By our analysis we found that the default occurs too quickly to be measured and no trend can be observed. The rule emphasized before has been revised. The new knowledge has been validated by an expert committee and the knowledge base has been modified. This process of rule validation is more challenging than generating rules with the datamining module. The next step of this prototype development is to better prepare data sets to find new relevant rules.

Being based on classical multivariate data statistical analysis algorithms, the data mining module must be fed with "good" data. This ensures users to discover new phenomena. For example, we can imagine that for some data sets, a process trend can be observed and for other data set, no trend can be observed. The main problem is then to master the domain range of data and to link it with information inferred by datamining algorithms.

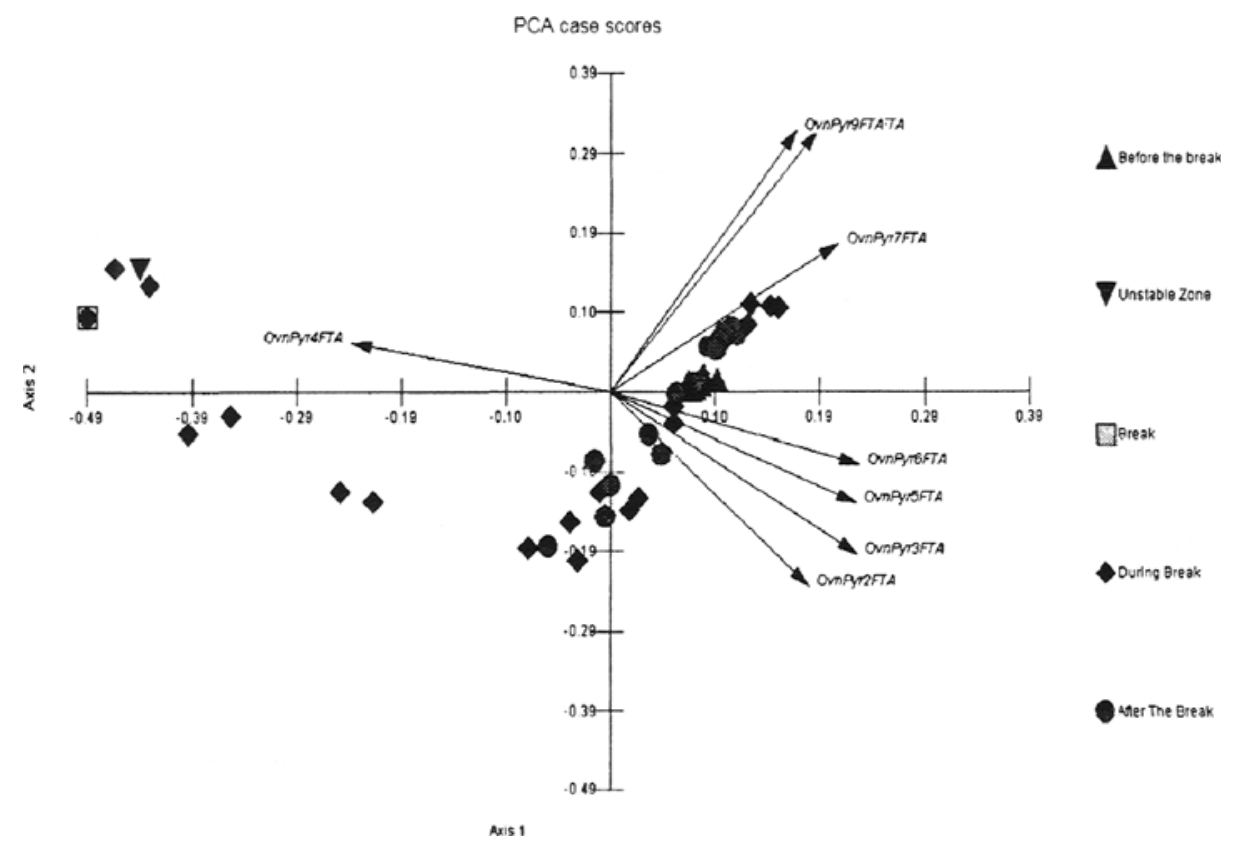

vector scaling 060

Figure 6 - The PCA of a Process Variable Set and its Correspondent Clusters Analysis. 


\section{CONCLUSION}

We achieve our goal of conceptualization and open the way for building a full featured product in a particular industrial area, with the manufacturing intelligent supervision system prototype and the advanced application development process. Connecting different concepts, this work approach can be called "research integration". This prototyping allows us to apply "basic" and "basis" concepts detailed during the conceptualization work. The conceptual framework, applying a balance between knowledge, technology and human management approach, allows us to exceed constraints linked to industrial developments and therefore allows us to exploit some scientific issues and richness of a specific industrial field.

\section{REFERENCES}

[Agrawal \& Srikant, 2000] Agrawal R, Srikant R, Fast Algorithms for Mining Association Rules, IBM Almaden Research Center, 2000

[Bassetto 2001], Bassetto S. Master thesis dissertation, 2001 @®ENSAM

[Beauvois \& al. 1987] Beauvois J.-L, Joule R.-V. Petit traité de manipulation à l'usage des honnêtes gens. PUG publishing, 7rd Ed, 1987.

[CLIPS, 1998], CLIPS 6.10 beginner and advanced Programming Guide, NASA, 1998.

[Ermine \& al. 1996], Ermine, JL., Chaillot, M., Bigeon P., Charreton B, Malavieille, D. La méthodologie MKSM, Cahier de lecture du MCX/APC n , 1996

[Ermine, 1996] Ermine J.-L. : Les systèmes de connaissances, Hermès Publishing , Paris, 1996.

[Faure \& Bisson, 2000] Faure, A. Bisson, G. Gérer les retours d'expériences pour maintenir une mémoire métier, étude chez PSA Peugeot Citroën, INRIA \& PSA Peugeot Citroën 2000

[PEUGEOT - PSA CITROEN, 1999] PEUGEOT PSA CITROEN Direction de la qualité et de l'innovation, Guide de créativité, 1999

[Jacqueson \& al. 2001] Jacqueson, D. Millet, S. Minel, A. Aoussat, Dynamique des connaissances en conception : acquisition, capitalisation et réutilisation, CPNI, PRIMECA 2001.

[Lefébure \& al, 2001] Lefébure R. and Venturi G., Datamining, Gestion de la relation client, personnalisation de sites web, Eyrolles publishing, 2nd Ed. 2001.

[Morin, 1990] Morin, E. Introduction à la pensée complexe, Paris, ESF Publishing, 1990.

[Napoli, 1997], Napoli, A. une introduction aux logiques de description, RR 3314 INRIA, 1997

[Sudipto, G. and al. 1998], Sudipo Guha, Rajeev Rastogi, Kyuseok Shim, CURE an efficient Clustering Algorithm for Large Databases, Stanford University \& Bell Laboratories, 1998

[Szymkowiak \& al., 2001], Szymkowiak, A. Larsen J., Hansen L.K. Hierarchical Clustering for Datamining , Technical University of Denmark, 2001.

[Terwiesch and Loch, 2002], Terwiesch C., Lock, C.H. Collaborative Prototyping and the pricing of customized products, The Warton School, R\&D INSEAD, 2002.

[Vernadat, 1996] Vernadat, F. Entreprise Modelling and Integration, Chapman \& Hall Publishing, 1996 